\title{
Experimental test of Bayesian saccade targeting under reversed reading direction
}

\author{
Johan Chandra ${ }^{1} \cdot$ André Krügel $^{1} \cdot$ Ralf Engbert $^{1}$ \\ Published online: 25 July 2019 \\ (C) The Psychonomic Society, Inc. 2019
}

\begin{abstract}
During reading, rapid eye movements (saccades) shift the reader's line of sight from one word to another for high-acuity visual information processing. While experimental data and theoretical models show that readers aim at word centers, the eye-movement (oculomotor) accuracy is low compared to other tasks. As a consequence, distributions of saccadic landing positions indicate large (i) random errors and (ii) systematic over- and undershoot of word centers, which additionally depend on saccade lengths (McConkie et al. Visual Research, 28(10), 1107-1118, 1988). Here we show that both error components can be simultaneously reduced by reading texts from right to left in German language $(N=32)$. We used our experimental data to test a Bayesian model of saccade planning. First, experimental data are consistent with the model. Second, the model makes specific predictions of the effects of the precision of prior and (sensory) likelihood. Our results suggest that it is a more precise sensory likelihood that can explain the reduction of both random and systematic error components.
\end{abstract}

Keywords Eye movements and reading · Bayesian modeling

\section{Introduction}

Most reading theories assume that readers aim at word centers, where visual information can be extracted optimally. The optimal viewing position (OVP) refers to the location within a word where refixation probability is the lowest (O’Regan \& Lévy-Schoen, 1987; Rayner, 1998; Reilly \& O'Regan, 1998). However, the performance of the oculomotor system during reading, as reflected in the statistics of within-word fixation landing positions, turns out to be unexpectedly imprecise since most of the observed fixations initially do not land on word center, but slightly left of it. This off-center position was termed the preferred viewing position (PVP) by Rayner (1979). The saccadic landingposition errors represent an important source of variability

Electronic supplementary material The online version of this article (https://doi.org/10.3758/s13414-019-01814-4) contains supplementary material, which is available to authorized users.

Johan Chandra

johan.chandra@uni-potsdam.de

1 Department of Psychology \& Cognitive Science Program, University of Potsdam, Am Neuen Palais 10, 14469 Potsdam, Germany in the reading process (Rayner, 1998). The current study is designed to investigate how the change in reading direction, i.e., German texts are read from right to left, can influence the oculomotor accuracy. We expect that the change in reading direction would interrupt readers' highly automated oculomotor control processes and, at the same time, could increase the precision of saccadic landing position. This manipulation also represents an interesting case for testing a recently developed Bayesian model of saccade planning (Engbert \& Krügel, 2010), which makes explicit assumptions about the origin and the amount of random and systematic errors within the oculomotor system during reading.

Since the seminal work of McConkie and colleagues (McConkie et al., 1988), it is well known that saccades in reading inhere two different kind of errors: random and systematic components. The random error component is assumed to reflect random perceptuo-oculomotor inaccuracy in the execution of saccades leading to the observation of broad Gaussian landing-position distributions within words in reading. The systematic range-error component is interpreted as a fundamental tendency of the oculomotor system to generate saccades of an average length leading to systematic overshoots of close word centers and systematic undershoots of distal word centers. Similar effects were reported in simple saccade targeting tasks (Kapoula, 1985; Kapoula \& Robinson, 1986). The systematic error can 
be statistically approximated by a linear landing-position function (Radach \& McConkie, 1998),

$\triangle_{P V P}=\lambda \cdot\left(L_{0}-L\right)$,

in which the term $\left(L_{0}-L\right)$ represents the deviation of saccades current launch-site distance to the center of the target word, $L$, from an optimal distance $L_{0}$ at which saccades during reading land on average at the word center. For $L$ larger than $L_{0}$, negative values of $\Delta_{P V P}$ represent the negative displacement of saccades' landing positions from the word center, i.e., a systematic undershoot. Positive values of $\triangle_{P V P}$, i.e., a systematic overshoot of word centers, result from launch-site distances $L$ smaller than $L_{0}$. The slope parameter $\lambda$ reflects the strength of the systematic error component. An estimated slope of $\lambda \approx$ 0.5 was observed in readers of English (McConkie et al., 1988) and German (Nuthmann et al., 2005), meaning that for each letter increment of the launch-site distance $L$, the means of within-word landing positions shift leftward with a magnitude of half a character space. Note that in the context of the current paper, random and systematic error components are completely independent according to McConkie et al.'s (1988) model.

Based on the framework of Bayesian integration in sensorimotor control (Körding, 2007; Körding \& Wolpert, 2004), Engbert and Krügel (2010) proposed a processoriented model of Bayesian saccade planning during reading that can be used to predict the size of random and systematic oculomotor errors in reading under altered reading conditions. According to the model, saccadic errors are the result of estimating target positions, i.e., the location of word centers, based on uncertain sensory information, and learned prior knowledge. Following Bayes' rule, given a noisy sensory observation of the word center at $x_{0}$, the optimal estimate of the true position $x$ can be calculated as the conditional posterior probability $\pi\left(x \mid x_{0}\right)$ according to

$\pi\left(x \mid x_{0}\right) \sim q\left(x_{0} \mid x\right) p(x)$,

where $p(x)$ denotes the learned probability distribution of target locations during reading (prior), i.e., the readers internal representation of the statistics of the low-level text properties such as word lengths and distances to target words. The conditional probability $q\left(x_{0} \mid x\right)$, called likelihood, reflects the sensory likelihood of the observation at position $x_{0}$ given a word center at $x$, i.e., uncertainty of the sensory measurement of the word centers depending on the visual input and other factors such as the allocation of attentional resources and the time available for the sensory measurements. By assuming that the prior and likelihood densities follow the Gaussian distribution function, the posterior distribution of saccadic random and systematic errors can be calculated analytically.
Random saccadic errors are reflected in the variance of the Gaussian posterior distribution, $\sigma_{P}^{2}$, which increases with increasing variance of the sensory likelihood $\left(\sigma_{0}^{2}\right)$ or the prior $\left(\sigma_{T}^{2}\right)$ or decreases with decreasing variance of the likelihood or prior distribution:

$\sigma_{P}^{2}=\frac{\sigma_{T}^{2} \sigma_{0}^{2}}{\sigma_{T}^{2}+\sigma_{0}^{2}}$.

Furthermore, Engbert and Krügel (2010) demonstrated that the systematic error component of saccades in reading, i.e., the launch-site contingent over- and undershoot of word centers, resulted from the shift of the posterior word-center estimate towards the prior distribution: Saccades overshoot word centers near the maximum of the prior distribution and systematically undershoot more distal word centers. Most importantly for the current study, the strength of the systematic error as reflected in the parameter $\lambda$ in Eq. 1, depends on the relative uncertainty of the prior and the sensory likelihood and can be calculated from the variances of the prior and the likelihood:

$\lambda=\frac{\sigma_{0}^{2}}{\sigma_{0}^{2}+\sigma_{T}^{2}}$.

Interestingly, unlike the effects on the random error component, prior and likelihood variances have opposite effects on the systematic errors of saccades: Reduced observational uncertainty (signaled by a decreased likelihood variance) will result in a reduced systematic error component (i.e., decreased $\lambda$ ) but reduced prior variance will result in an increased systematic error component.

Here we assumed that reading against the normal reading direction would interrupt the highly automatic process of saccade planning and induce a more careful strategy of saccade control. We also assumed that the more careful saccade planning is associated with attenuated internal sensory noise (i.e., reduced likelihood uncertainty) achieved by certain mechanisms such as by increasing the time needed to plan a saccade and/or by allocating more visualattentional resources to measure target positions. On the other hand, as the low-level properties of the text (word length statistics and distance to target words) were largely preserved in the reversed reading direction conditions, we expected that readers' prior belief on word-length statistics would remain largely unaffected.

Based on this assumption, the Bayesian model (Engbert \& Krügel, 2010) makes very strong predictions: First, according to Eq. 3, reduced sensory uncertainty (i.e., reduced likelihood variance $\sigma_{0}^{2}$ ) should lead to reduced posterior uncertainty (i.e., reduced $\sigma_{P}^{2}$ ), hence, reducing the random errors of saccadic landing positions within words. Second, as reduced sensory uncertainty is also related to a reduction of the systematic error component in the Bayesian model (Eq. 4), we expect that reading texts from right to 
left should also lead to reduced systematic errors, reflected by a reduced parameter $\lambda$ of the linear landing positions function.

\section{Method}

\section{Study design and material}

Participants read an excerpt from the German version of the novel "The Adventure of the Empty House" (Doyle, 2009), which was broken into 600 lines (maximum of 85 character spaces per line). During the first eye-tracking lab session, participants read 200 lines of the excerpt in a typical direction from left to right (control condition). For the second and the third sessions, participants continued reading from the part where they left off in the previous session, but in atypical reading direction, from right to left (experimental condition). To minimize the influence of cognitive processes on within-word landing positions, individual words were presented normally, written from left to right, in all conditions with one exception: The word order in a sentence was inverted in experimental condition to match the reversed reading direction (e.g., the first word of a sentence begin on the right side of the screen; see Fig. 1).

\section{Participants and apparatus}

Analyses of our primary hypotheses are based on parameter estimates of fixations landed within 3- to 7-letter words and were launched from 1 to 5 characters away from the word beginning for each reading condition (left-to-right vs. right-to-left; all distributions contingent on word length and launch-site). Based on a large eye-movement corpus consisting of 275 adult readers (Potsdam Sentence Corpus;
Kliegl et al. (2006)) we analyzed the distributions of samples randomly drawn from 10,20 , and 30 participants. Using 100 independent repetitions per sample size, we estimated that 20 participants were sufficient to reproduce the estimated value of $\lambda=0.28$ reported by Krügel and Engbert (2010) with reliability better than 0.05 character units $(95 \%$ CI $[0.27,0.30])$. For the current study, we exceeded the estimated sample size by testing a total of 32 participants (27 females, five males; age range 19-38 years). After declaring their informed consent, participants took part in three 45-min eye-tracking lab sessions. They received a total of 30 euros or $3 \mathrm{~h}$ of course credit for their participation. Participants were all naive with respect to the purpose of the experiment and reported normal or corrected-to-normal vision. The experiment conformed to the Declaration of Helsinki.

With the head supported by a chin rest, participants read the text with a viewing distance of $70 \mathrm{~cm}$ in front of a 19-inch Mitsubishi Diamond Pro 2007 monitor (screen resolution $1280 \times 1024$ pixels, refresh rate $100 \mathrm{~Hz}$ ). The stimuli (font: Courier, size: 18 pixels, color: black) were presented at the vertical center line of the computer display with grey background color. Eye movements were recorded binocularly using an EyeLink 1000 System (SR Research, Osgoode/Ontario, Canada) with a sampling rate of 1000 $\mathrm{Hz}$ and spatial resolution better than $0.01^{\circ}$. Participants had to answer three questions related to the text they have just read at the end of each session. On average, two out of three questions were correctly answered on each session.

\section{Data preparation}

Saccade detection and data exclusion criteria. Raw data were marked if they contained blinks. Only trials with

\section{Control condition:}

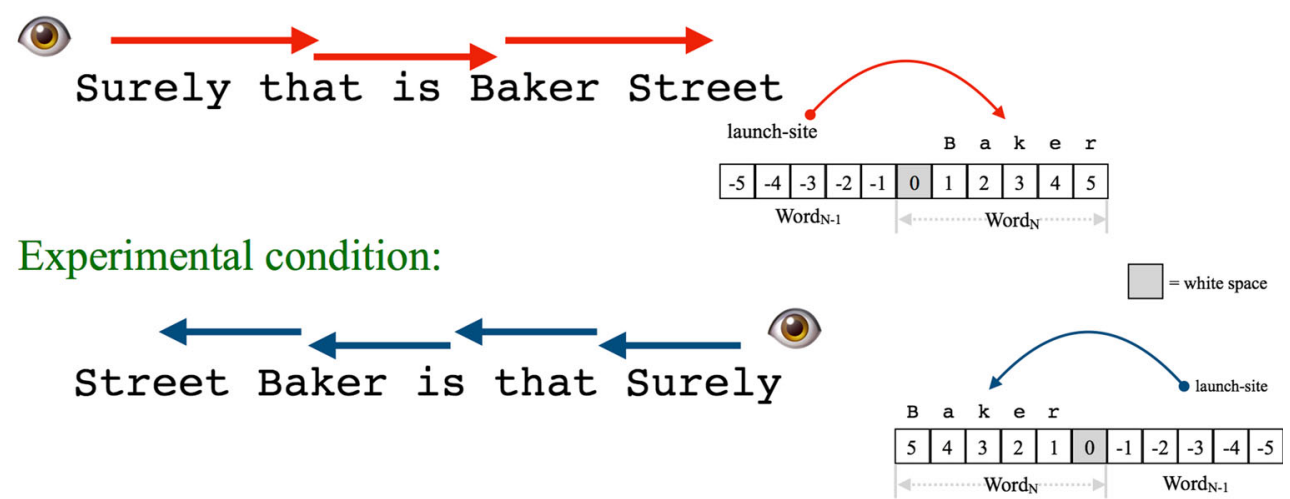

Fig. 1 Example of experimental manipulation. Normal reading in control condition (a). In experimental condition (b), the sentence is read from right to left and incoming saccades on target $\operatorname{word}_{N}$ are calculated from the right. Letter position is coded in the global reading direction beginning with the space before the word that is denoted as letter position 0. Red and blue arrows represent saccades during reading in control and experimental conditions, respectively 
durations of more than $1000 \mathrm{~ms}$ before the first detected blink were included in saccade detection procedure. Fixations and saccades information was obtained using a velocity-based algorithm developed by Engbert et al. (2003). A total of 307,908 fixations were detected. For further analysis, fixations landing on the first and last words of a sentence, as well as the first and last fixations in a trial, were excluded. Furthermore, fixations with short (less than $20 \mathrm{~ms}$ ) and long (greater than $1000 \mathrm{~ms}$ ) durations, fixations landing outside the text area and fixations following saccades within a character unit were also removed from the analysis. Trials containing fixations longer than 2000 $\mathrm{ms}$ (above 95\% of the distributions) or less than three fixation points were excluded from analysis. This resulted in 166,265 valid fixations (see Online Supplemental Material for summary).

Landing-position distributions. All analyses on saccadic within-word landing positions are based on progressive word-to-word saccades during first-pass reading. As a first step, saccades of each session (one control, two experimental sessions) were grouped based on word length (3-7 letters) and launch-site distance (-1 to -5 characters), resulting in 25 word-length and launch-site-specific data sub-samples per session. For each sub-sample, the relative frequency of saccadic landing positions on each letter within the fixated word was calculated. Next, to estimate the means and standard deviations of the distribution of landing positions, truncated Gaussian curves were fitted using Bayesian parameter inference (Kruschke, 2014) available in the package rjags (Plummer, 2016) in the $\mathrm{R}$ environment (see Online Supplement for more details on the fitting procedure).

Fixation duration and fixation probability measures. For first-pass reading data, we computed different fixation duration measures. Single fixation duration (SFD) refers to the time spent on a word which received exactly one fixation during first-pass reading. When a word received more than one fixation in first-pass reading, the duration of the first fixation generated the first of multiple fixations duration measure (FMD). Total viewing time reflects the sum of all fixations on a word. For word-length effect analysis, fixation duration measures were calculated separately for words of length 3-11 characters. Since fixations landing on a word could come from different saccade types, we computed the word-based fixation probabilities for wordto-word saccades, word skipping, refixation, and regression during the first-pass reading. Regression cases were counted for words whose saccade directions went against global reading directions, right to left for control condition and vise versa for experimental conditions, and landed on previously read words. For refixation cases, a word was refixated if it received two or more fixations consecutively, regardless the direction of the saccades.

\section{Results}

Before we present our results on saccadic landing positions, we first report the statistics of fixation duration and fixation probability measures as an overview of the global reading performances when texts were read normally and when they were read against the normal reading direction. Then, we investigate the random and systematic saccadic errors under both reading conditions.

\section{Fixation duration and fixation probability}

Since words were presented in their typical layout (written from left to right) in both conditions, we expected only moderate effects of changing the global reading direction (word order from right to left) on readers' fixation duration and fixation probability measures. If not otherwise mentioned, the results refer to the comparisons between the first experimental session and the control condition (normal reading).

On global level, readers' average fixation duration was $38 \mathrm{~ms}$ longer when texts were read from right to left compared to normal reading direction. This general prolongation in reading texts from right to left holds true across different types of fixations duration measures (single-, first-, second-of-multiple fixation durations, and total viewing time) as a function of word length. For example, the estimated means of fixation duration for words with medium (5-7 characters) length were $25-55 \mathrm{~ms}$ longer for all fixation types. However, as visualized in Fig. 2, fixation duration measures as a function of word lengths in the experimental condition do not qualitatively differ from those observed in the control condition.

Moreover, we found a reduced rate of word skippings and more refixations (i.e., saccades within the same word) when texts were read from right to left. For the control condition where texts are read normally from left to right, participants moved their eyes mostly from the currently fixated word to the next word (43\% of the saccades). A third of the saccades skipped the word next to the currently fixated word. About $16 \%$ of observed saccades moved within a word (refixations) and $13 \%$ went backward (regressive saccades). When texts were read from right to left, participants generated slightly more forward word-toword saccades $(49 \%$ in the first right-to-left session and $51 \%$ in the second session). However, skipping saccades were reduced to around $17 \%$ (in both right-to-left sessions) and readers refixated words more often $(20 \%$ and $22 \%$ of the time). Approximately the same proportions of regression saccades as in normal reading were observed (14\% and $11 \%$ of the saccades moved backwards in the right-to-left sessions). Thus, fixation probabilities for word-to-word saccades and regressive saccades were not 

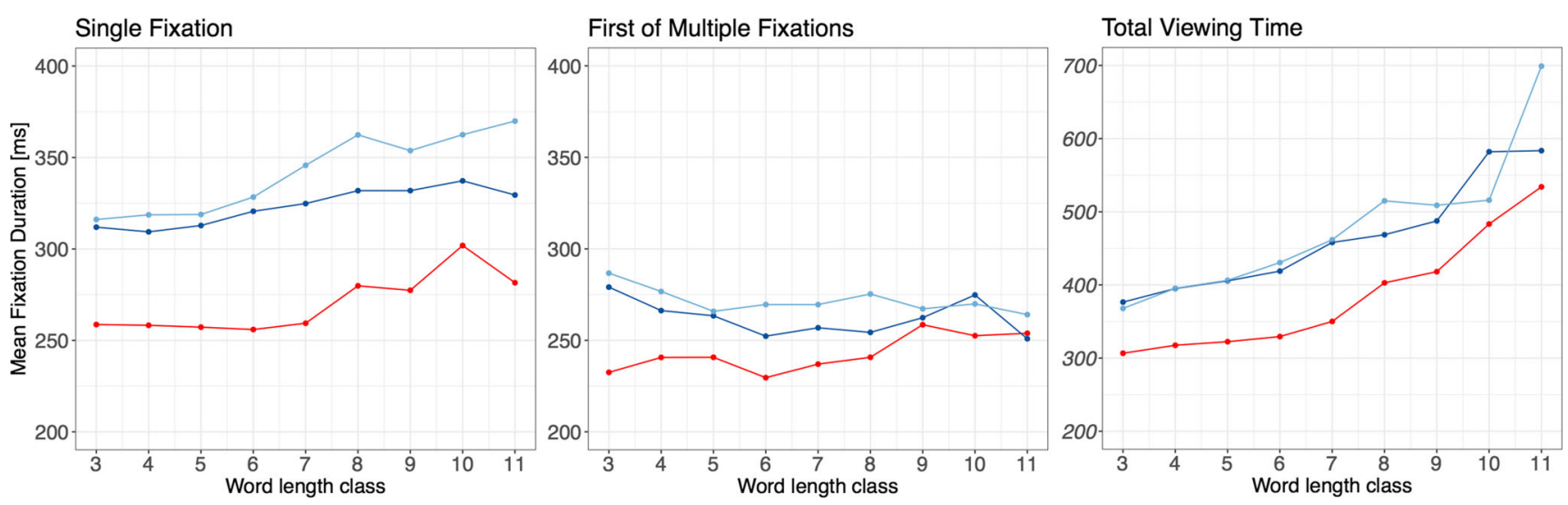

Fig. 2 Fixation duration measures as function of word length. Red lines and dots represents mean fixation durations observed in normal reading. Dark and light blue colors represent data observed in

experimental conditions. When texts were read from right to left, readers tend to fixate longer than when texts were read normally

affected by the change in reading direction. However, the increased refixation rate and decreased skipping rate in the experimental condition may indicate that reading against normal direction requires additional effort in saccade programming processes. More detailed global summary statistics on fixation durations and fixation probabilities and the results of linear-mixed model analysis on fixation duration measures are provided in the Online Supplemental Material.

\section{Landing position analyses}

\section{Empirical results}

The current study was designed to test the assumption that reading against the normal reading direction would modulate eye movement accuracy during reading. Therefore, we analyzed both the random error component (reflected by the variance of within-word landing distributions) and the systematic error component of saccades (reflected by the strength of the launch-site contingent shift of saccades mean within-word landing positions). Figure 3 shows the observed distributions of saccadic landing positions for all sessions and word-length by launch-site combinations along with the obtained truncated Gaussian fits. Figure 3 quickly reveals that, regardless of experimental condition, landing distributions are broad (random error component) and shift within words as a function of the distance of saccades' launch sites (systematic range-error component). However, even at a first glance, Fig. 3 suggests that distributions of saccadic landing positions in the right-to-left reading condition are narrower than the distribution in normal reading and that they seem to be more aligned to the word center. Parameter estimates (mean and SD) for each distribution are reported in the Online Supplemental Material.
For a detailed analyses of our hypotheses (reduced random errors and reduced systematic saccadic errors in right-to-left reading), separate analyses on the means and the standard deviations of the Gaussian saccadic landing

First, we looked at the effect of reading direction on the standard deviations of saccadic landing distributions within words. Figure $4 \mathrm{a}$ shows the standard deviations of the landing site distributions as a function of saccadic launch-site distances towards the centers of target words for each session; linear regression lines were fitted to the standard-deviation estimates. Saccadic landing positions are significantly narrower in the right-to-left reading condition compared to the normal left-to-right reading condition, confirming our first hypothesis that changing the reading direction reduces the random-error component in saccades. After conducting a linear regression model with (center-based) launch-site distance and session as predictor variables on landing-site standard deviation measures (see Table 1), we found that the difference between the standard deviations in the normal reading session and the first experimental session could be quantified as large as about 0.36 to approximately 0.4 character spaces on average. Furthermore, the random error component is modulated by the launch-site distance in both conditions. The modulation is stronger in the right-to-left reading condition than in the normal left-to-right reading condition. There is no difference between the first and the second experimental sessions.

Second, we analyzed the effect of reading direction on the strength of the systematic error component in saccades, i.e., the shifts of saccadic landing distributions as a function of saccadic launch-site distances towards word centers, and calculated the parameters of the linear landing position function using linear regressions. The distributions were conducted. 


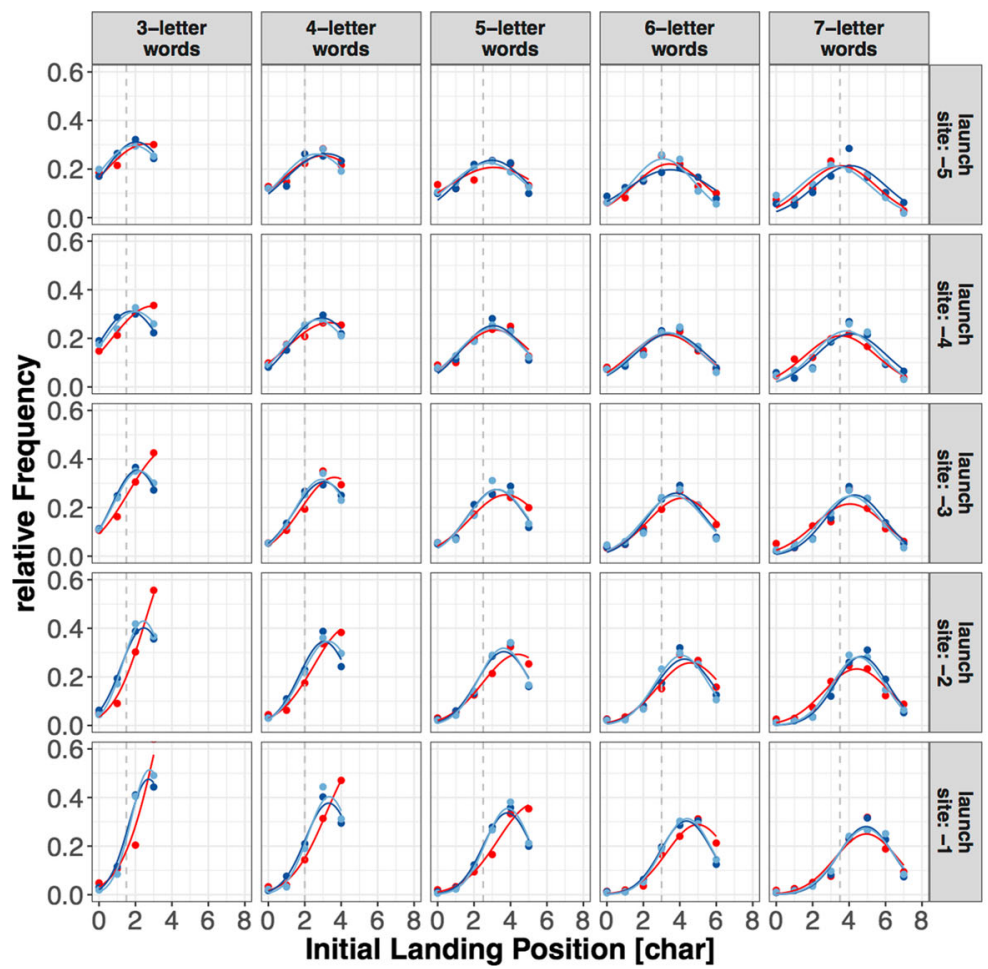

Reading direction

$\rightarrow$ Left-to-right

- Right-to-left (1st)

$\rightarrow$ Right-to-left (2nd)

Fig. 3 Within-word landing-position distributions of progressive word-to-word saccades based on word-length and launch-site distance. Red color represents data observed in control condition (normal left-to-right reading) and blue hues represent data observed in experimental conditions (right-to-left reading)

strength of the systematic error component is reflected in the parameter $\lambda$ of Eq. 1, i.e., the slope of the regression line. According to our predictions based on Engbert and Krügel's
(2010) model of Bayesian saccade planning, we expected a reduced slope of the linear landing position function when reading from right to left. Figure $4 \mathrm{~b}$ shows that our results

\section{a) Random error component}

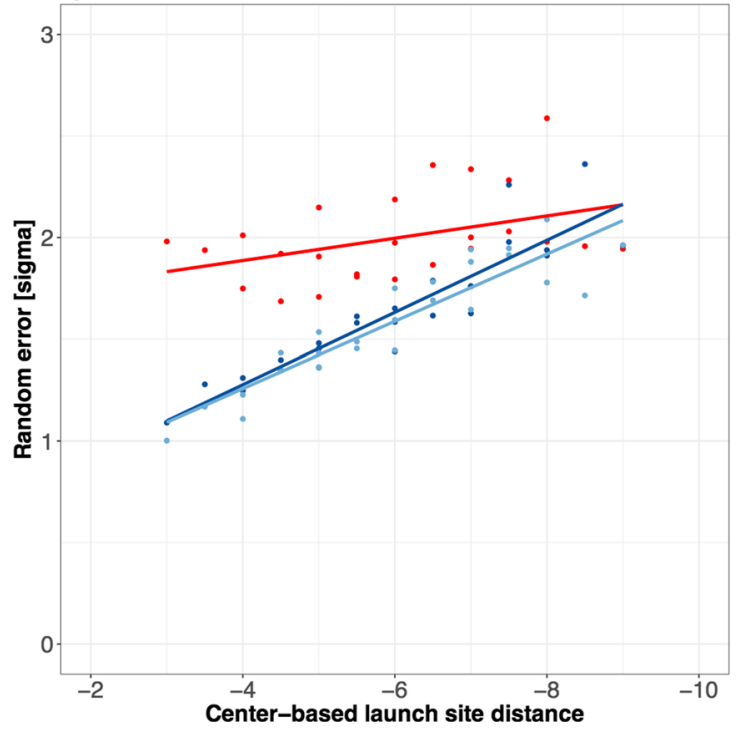

Fig. 4 a Random error component: Estimated standard deviations are plotted against center-based launch-site distance to word center. b Mean landing positions relative to word center are plotted against launch-site distance to word center. The slope of the linear regression b) Systematic error component

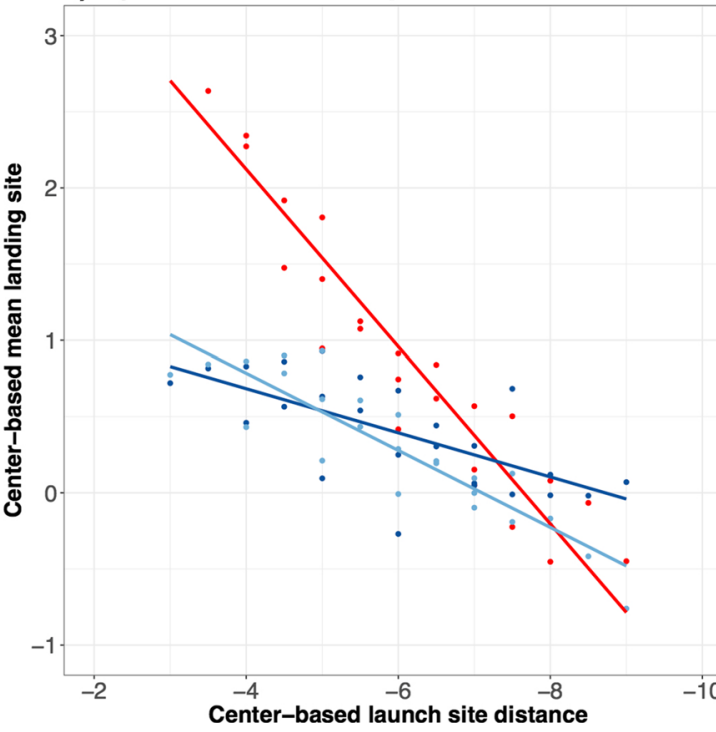

line represents the strength of the launch-site effect, i.e., the systematic error component. Red color represents data from normal left-to-right reading. Blue hues represent right-to-left reading 
Table 1 Regression results for the standard deviation and mean of within-word landing position distributions

Dependent variable:

Standard deviation

(1)

$$
\begin{aligned}
& -0.055^{\mathrm{c}}(0.020) \\
& -0.365^{\mathrm{c}}(0.044) \\
& -0.409^{\mathrm{c}}(0.044) \\
& -0.123^{\mathrm{c}}(0.028) \\
& -0.110^{\mathrm{c}}(0.028) \\
& 1.997^{\mathrm{c}}(0.031) \\
& 75 \\
& 0.777
\end{aligned}
$$

Right-to-left (1st)

Launch site : Right-to-left (1st)

Launch site : Right-to-left (2nd)

Constant

Observations

Adjusted $R^{2}$
Mean landing position

(2)

$0.582^{\mathrm{c}}(0.033)$

$-0.565^{\mathrm{c}}(0.075)$

$-0.681^{\mathrm{c}}(0.075)$

$-0.437^{\mathrm{c}}(0.047)$

$-0.329^{\mathrm{c}}(0.047)$

$0.959^{\mathrm{c}}(0.053)$

75

0.864

Note: Standard errors are reported in parentheses. ${ }^{\mathrm{a}} \mathrm{p}<0.1 ;{ }^{\mathrm{b}} \mathrm{p}<0.05 ;{ }^{\mathrm{c}} \mathrm{p}<0.01$

meet the prediction: Changing the reading direction leads to a dramatically reduced systematic shift of the landing positions. Table 1 (right panel) shows the parameters of the regression model. In the normal left-to-right reading condition we observed a rather strong shift of mean landing positions (covariate 'Launch site': $\beta=\lambda=0.58, p<$ 0.001 ). However, when texts are read from right to left, the slope of the linear landing position functions decreases to $\lambda=0.15$ in the first right-to-left reading session (covariate 'Launch site : Right-to-left (1st)': $\beta=-0.44, p<0.001$ ) and to $\lambda=0.25$ in the second right-to-left session (covariate 'Launch site : Right-to-left (2nd)': $\beta=-0.33, p<0.001$ ).

Taken together, reversing the reading direction generates strong reductions of saccadic errors both in terms of the random fluctuations of landing positions within words, as well as in terms of the systematic over- and undershoots of word centers.

\section{Model simulation}

In order to check the interpretations of the results, we aimed at demonstrating that a Bayesian model of saccade planning is able to simultaneously account for the observed effects on saccades' random and systematic error components during reading for both the normal left-to-right reading condition and the reversed reading condition. Following Engbert and Krügel (2010), we defined a Bayesian model based on the assumption of Gaussian prior- and likelihood probability densities (prior: $p(x)=\mathcal{N}\left(x ; \mu_{T}, \sigma_{T}^{2}\right)$; likelihood: $\left.q\left(x_{0} \mid x\right)=\mathcal{N}\left(x ; x_{0}, \sigma_{0}^{2}\right)\right)$ and also adopted the assumption that sensory observations of the word center are generally unbiased, i.e., that the maximum of the likelihood, $x_{0}$, is aligned to the center of the target word. According to our assumption that readers employ the same prior in all reading conditions, we applied the two free parameters $\mu_{T}$ and $\sigma_{T}^{2}$, i.e., the mean and the variance of the prior distribution, to all reading conditions in the same way. With respect to the likelihood, we assumed that the variance $\sigma_{0}^{2}$ increases linearly with increasing distance $x_{0}$ of the word center from the starting position of the saccade, i.e., $\sigma_{0}^{2}=m x_{0}+b$. For simplicity and to avoid over-fitting, we assumed that the slope parameter $m$ is the same for both reading conditions. However, to match our central assumption that reading against the normal reading direction led to reduced sensory uncertainty, we employed different intercept parameters for reading from left to right, $b_{l r}$, and reading from right to left, $b_{r l}$. Thus, our model included five free parameters, i.e., the mean and standard deviation of the prior $\left(m u_{T}\right.$ and $\sigma_{T}^{2}$ ), the slope parameter $m$ and the intercept parameters $b_{l r}$ and $b_{r l}$ to calculate the variance of the likelihood density $\sigma_{0}^{2}$ for left-to-right reading or right-to-left reading, respectively. By using a Nelder-Mead simplex (directsearch) method (MATLAB; The MathWorks, Natick, MA, USA), we fitted the free parameters of the Bayesian model by minimizing the sum-of-squares error (SSE) between the posterior probability estimates predicted by the model and the observed landing-position distribution from the control condition (reading from left to right) and the first session in the right-to-left reading condition. For the prior, we obtained a highly plausible mean of $\mu_{T}=7.71$ and a variance of $\sigma_{T}^{2}=2.77$. For the likelihood, we obtained a slope parameter of $m=0.26$, meaning that the variance of the likelihood in the model increases by 0.26 character spaces for each one-letter increment of the distance between the launch site and the word center. Most importantly, we obtained highly different intercept estimates for the calculation of the sensory uncertainty $\sigma_{0}^{2}$ in the different reading conditions. According to our hypotheses, the estimated intercept parameter for reading against the normal reading direction is more than three times smaller than the estimated intercept for the normal left-to-right reading condition: $b_{r l}=0.45<b_{l r}=1.39$. 


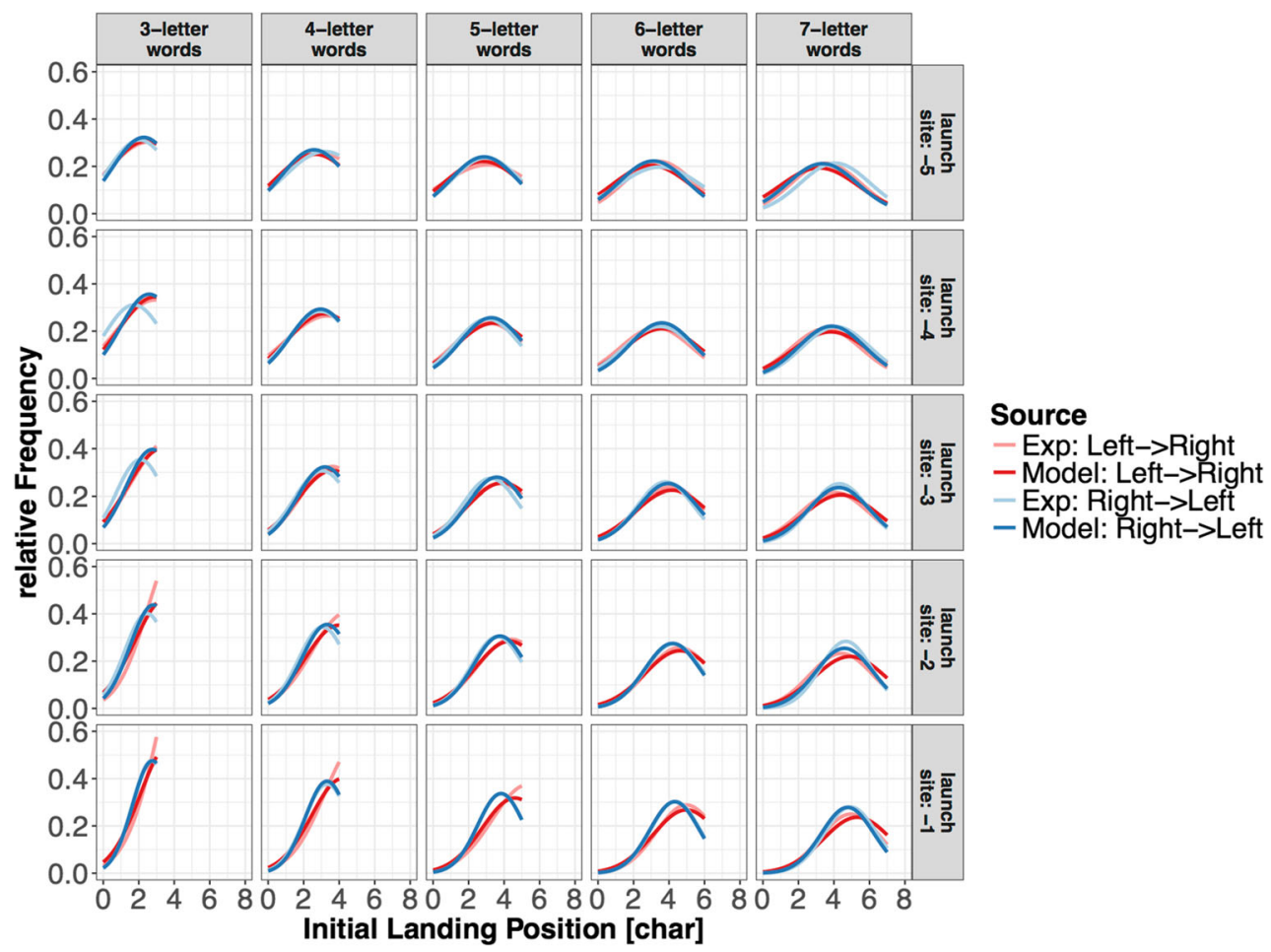

Fig. 5 Predicted posterior estimates and observed landing distributions. Model prediction is shown in dark colors, experimental observations in light colors. Red curves represent normal right-to-left reading condition and blue curves represent data and predictions from right-to-left reading

Figure 5 reveals a very good fit of the posterior estimates of word centers for different word length and launch-site distances as predicted by the Bayesian saccadeplanning model and the landing-position distributions obtained in the reading experiment. Obviously, the model is capable in capturing both the narrower and more- word-center-aligned distributions in the right-to-left reading conditions compared to the distributions observed in the normal left-to-right reading.

More specifically, Fig. 6 visualizes the goodness-of-fit of the model simulations with respect to the predicted random and systematic saccadic errors when reading either under a) Random error component

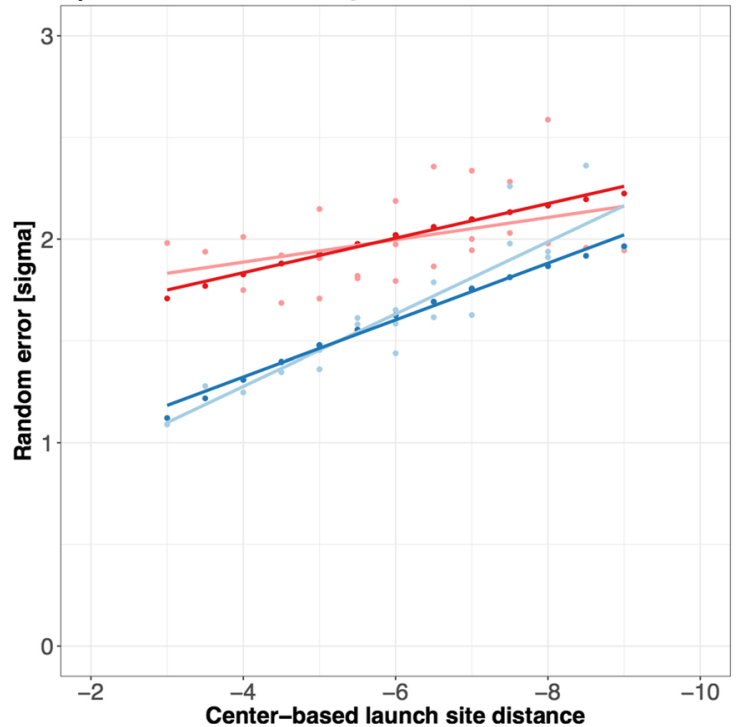

b) Systematic error component

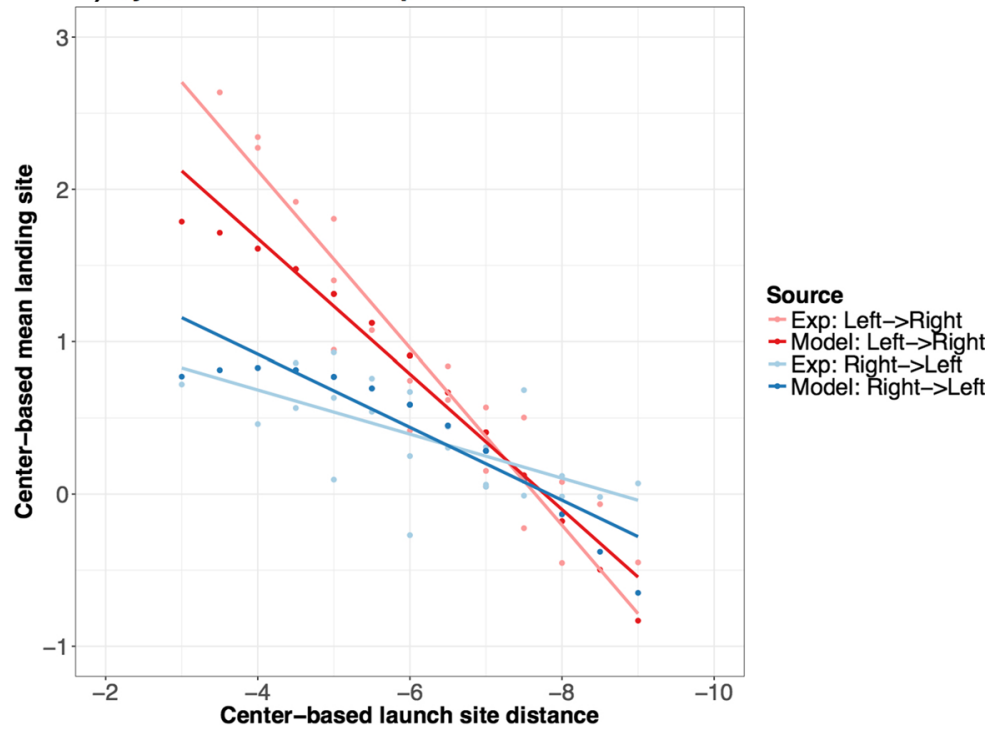

Fig. 6 Predicted posterior estimates and observed landing distributions. Model predictions are shown in dark colors, experimental observations in light colors. Red curves represent normal right-to-left reading condition and blue curves represent data and predictions from right-to-left reading 
normal conditions or against the normal reading direction. First, as shown in Fig. 6a, the model is able to reproduce the strong reduction in the random-error component of saccades as reflected in the reduced standard deviations of withinword landing position distributions. At the same time, the model also accurately predicts the reduced systematic range-error component as reflected in the decreased slope of the linear landing-position function for reading text against the normal reading direction (see Fig. 6b).

Taken together, both the experimental data observed in the reading experiment and the numerical simulations of the Bayesian saccade planning model lend support to our assumption that reading against the typical reading direction enhances eye-movement accuracy brought about by a reduction of sensory uncertainty in the observation of the word center during saccade planning.

\section{Discussion}

In summary, our results demonstrate that reading texts in non-standard direction enhances eye movement accuracy. Saccades in reading inhere large random and systematic errors (McConkie et al., 1988), however, it is largely unknown whether these errors can be reduced. Furthermore, according to McConkie et al.'s model, there is no coupling between random and systematic error components, i.e., the model does not make the corresponding prediction that can be tested experimentally. During reading, visuo-motor and language-related processes compete for visual and attentional resources and, obviously, the observed extent of oculomotor inaccuracies in normal reading do not generally obstruct the highly efficient reading process of experienced readers. Thus, it is interesting to know whether the observed accuracy of saccades in reading reflects a fundamental accuracy limit or whether saccades in reading are only as accurate as necessary, possibly in favor of reduced resources use. Our results reveal that both saccadic error components are substantially reduced when texts are read against the normal reading direction, which clearly demonstrates that the oculomotor system is able to generate saccades with better precision and accuracy during reading. One might argue that globally shorter saccades generated under the experimental condition could be a potential explanation for the observed improved saccade targeting process. However, such an explanation is in contradiction with two points: First, the average forward saccade amplitude in the experimental condition is only one character shorter than the similar saccade amplitude observed in the control condition. These relatively shorter saccades seem to be the result from generally less skipping and/or more refixation saccades generated in the experimental condition. Second, the main analysis on the within-word landing position distributions in this study was conducted on word-to-word forward saccades only-with similar lengths for both conditions. Although there might be a different number of observations across the two conditions, effects from other saccade types can be excluded from our analysis.

More specifically, we found that readers' eye movements were less variable when reading against the normal reading direction. We estimated a drop of the standard deviation of saccadic landing distributions from about 1.6 character spaces in the standard left-to-right reading condition to approximately 0.6 character space in the right-to-left reading condition. However, this effect was modulated by the distance of the center of the target word from saccades starting positions. The effect was the largest for short distances of word centers and was marginalized for distances larger than eight letters, suggesting that the improvement of saccadic accuracy during reading might be limited to a word-center-based window of eight letters in the reading direction. Nonetheless, as it is widely accepted that in reading, word centers are computed based on information about word boundaries, i.e., the spaces before and after the words (Rayner, 1998; Krügel \& Engbert, 2014), it is very likely that the effective window size for accuracy enhancements reaches 3-4 letters further into the periphery. Interestingly, this agrees well with the finding that readers obtain word-length information within a window of at least 12-15 letters from fixation position in reading direction reported by McConkie and Rayner (1975).

Even more importantly, the systematic shift of landing positions within words as a function of saccades' launch sites (the launch-site effect) was also largely reduced from a shift of slightly more than half a character for each one-letter increment of the launch-site distance in normal reading $(\lambda=0.58)$ to 0.15 and 0.25 characters in the right-toleft reading conditions. This is particularly remarkable as it has been shown that the launch-site effect during reading is very robust (McConkie et al., 1988; Nuthmann et al., 2005; Reilly \& O'Regan, 1998).

Our results can be very well interpreted against the backdrop of a recently developed Bayesian saccade planning model (Engbert \& Krügel, 2010) as demonstrated by the model simulations. The model replicates the simultaneous decrease in random and systematic error components and achieves an excellent goodness of fit. Our simulations demonstrate that the results are compatible with the assumption that readers in the experimental condition obtain more precise sensory information during saccade planning than those who read the texts normally from left to right. Why should this be the case? First of all, it is highly plausible that a reversal of the reading direction interrupts the otherwise highly automated saccade planning processes during reading and results in a more deliberately goal-oriented saccade control. A possible mechanism to 
explain the finding is a change in the distribution of covert attention, which can lead to increased contrast sensitivity and noise reduction in the periphery (Carrasco, 2011). Nonetheless, obtaining more precise sensory information must come at a cost, as reflected in the increased fixation durations during the reading from right to left condition. It remains open, however, whether these increased fixation durations are directly attributable to a more precise saccade planning in general, or whether they reflect an increased lexical difficulty (e.g., due to uninformative parafoveal information) in the right-to-left reading condition or a combination of both. Thus, it is also possible that saccade control indirectly benefits from the increased reading difficulty, which results in more time to obtain the more precise sensory information before the execution of the next saccade program.

Next, it is worth considering that a simultaneous reduction of random and systematic errors is not trivial. In the range-error model proposed by McConkie et al. (1988), random and systematic saccadic errors are conceptualized as independent error components. In the Bayesian saccade planning model proposed by Engbert and Krügel (2010), however, they are inherently linked to the precision of readers' prior knowledge and the sensory likelihood. Interestingly, according to Eq. 4, reduced systematic errors of saccades can be the result of either increased precision of the sensory likelihood or decreased precision of the prior. On the other hand, decreased precision of the prior would inflate the random saccadic errors according to Eq. 3. Thus, a simultaneous decrease of both error components as observed in our reading experiment can only result from increased precision of the sensory information.

The fact that the pattern of saccadic errors in reading is typically considered as highly robust is further reflected in most computational reading models, such as the EZ Reader model (Reichle et al., 2003), SWIFT (Engbert et al., 2005; Schad \& Engbert, 2012), Glenmore (Reilly \& Radach, 2006), or SERIF (McDonald et al., 2005), which incorporate the random and systematic errors of saccades based on the mathematical formulation and the numerical values reported by McConkie and colleagues (McConkie et al., 1988). However, our results show that the oculomotor system is highly adaptive and the saccadic errors can be modulated during reading to a surprisingly large extent. This emphasizes that reading models could be improved by implementing process-oriented assumptions for the saccade-planning process. The Bayesian saccade planning model (Engbert \& Krügel, 2010) represents a good candidate to be integrated into these reading models. Additionally, both cognitive and oculomotor model parameters should be estimated simultaneously during model fitting.
In future studies, it would be interesting to see how different reading instructions (e.g., proofreading), which might demand for more precise saccade targeting during reading, could modulate the accuracy of eye movements, also for the standard left-to-right reading. Several studies have addressed the effects of reading instruction but with a strong focus on temporal aspects of eye-movement control (Radach et al., 2008; Kaakinen \& Hyönä, 2010; Schotter et al., 2014). Radach et al. (2008) reported only minor effects of superficial versus reading-for-comprehension on saccades' global landing positions in words, but Kaakinen and Hyönä (2010) reported a left shift of landing positions under proofreading instruction. However, a comprehensive analysis of saccadic errors, both random and systematic, under different reading instructions is currently missing.

We close the discussion section by considering possible limitations of our experiment. Changing the reading direction might also affect the orthographic and lexical pre-processing of words that are next to the currently fixated word, since the relative positions of word beginnings and word endings are reversed in the right-to-left reading condition. Furthermore, the relationship between parafoveal preprocessing of words and saccades' initial landing positions within these words (the so-called cognitive landing site effects; Underwood and Radach (1998)) is much less clear. The literature on this issue reported controversial results, ranging from studies that argue against an effect of parafoveal preprocessing on within-word landing positions to studies reporting significant cognitive landing-site effects during reading (see Vonk et al. 2000, for a review]. However, it is fair to say that the studies which claimed to find the cognitive landing site effects reported effects that were extremely small (usually in the order of fractions of character units) compared to the effects observed in our experiment. Even more importantly, in a reading experiment where the saliency of word beginnings was manipulated (high and low initial trigram frequency), Vonk et al. (2000) found a small constant shift of landing positions by 0.3 character units to the left in words with low-frequency beginnings. The authors also analyzed saccades landing positions contingent on saccades launchsite distances but found no effect of the saliency of word beginnings on the systematic error component. Therefore, it is unclear whether the reversal of word beginnings and word endings in the reversed reading condition contributes to the observed reductions in saccadic random and systematic errors in our experiment, but we assume that such an influence would be small.

Acknowledgements This work received support by the Deutsche Forschungsgemeinschaft (DFG, German Research Foundation), Grant EN471/15-1 and project number 317633480-SFB 1287, Project B3. 
Author contributions R. Engbert and A. Krügel developed the study concept and the study design. Testing and data collection were performed by J. Chandra. J. Chandra and A. Krügel performed the data analysis and all authors contributed to the interpretation. J. Chandra and A. Krügel drafted the manuscript, and R. Engbert provided critical revisions. All authors approved the final version of the manuscript for submission.

Open practice statement Neither of the experiments reported in this article was formally preregistered. Neither the data nor the materials have been made available on a permanent third-party archive; requests for the data or materials can be sent via e-mail to the lead author at johan.chandra@uni-potsdam.de.

\section{References}

Carrasco, M. (2011). Visual attention: The past 25 years. Vision Research, 51(13), 1484-1525.

Doyle, A. C. (2009). The adventure of the empty house (A. Wlk trans). Alexander Wlk. (Retrieved from http://gutenberg.spiegel.de/buch/ das-leere-haus-1153/1. (Original work published 1903)).

Engbert, R., \& Krügel, A. (2010). Readers use Bayesian estimation for eye movement control. Psychological Science, 21(3), 366-371.

Engbert, R., Nuthmann, A., Richter, E. M., \& Kliegl, R. (2005). Swift: a dynamical model of saccade generation during reading. Psychological Review, 112(4), 777.

Engbert, R., Sinn, P., Margenthaler, K., \& Trukenbord, H. (2003). Microsaccade toolbox. http://read.psych.uni-potsdam.de

Kaakinen, J. K., \& Hyönä, J. (2010). Task effects on eye movements during reading. Journal of Experimental Psychology: Learning, Memory, and Cognition, 36(6), 1561.

Kapoula, Z. (1985). Evidence for a range effect in the saccadic system. Vision Research, 25(8), 1155-1157. Retrieved from http:// www.sciencedirect.com/science/article/pii/0042698985901051 https://doi.org/10.1016/0042-6989(85)90105-1

Kapoula, Z., \& Robinson, D. (1986). Saccadic undershoot is not inevitable: Saccades can be accurate. Vision research, 26(5), 735743.

Kliegl, R., Nuthmann, A., \& Engbert, R. (2006). Tracking the mind during reading: The influence of past, present, and future words on fixation durations. Journal of Experimental Psychology: General, 135(1), 12.

Körding, K. P. (2007). Decision theory: What "should" the nervous system do?. Science, 318(5850), 606-610. https://doi.org/10.1126/ science. 1142998

Körding, K. P., \& Wolpert, D. M. (2004). Bayesian integration in sensorimotor learning. Nature, 427(6971), 244-247. https://doi.org/10.1038/nature02169

Krügel, A., \& Engbert, R. (2010). On the launch-site effect for skipped words during reading. Vision Research, 50(16), 15321539. https://doi.org/10.1016/j.visres.2010.05.009

Krügel, A., \& Engbert, R. (2014). A model of saccadic landing positions in reading under the influence of sensory noise. Visual Cognition, 22(3-4), 37-41. https://doi.org/10.1080/13506285.2014. 894166

Kruschke, J. K. (2014). Doing Bayesian data analysis: A tutorial with R, JAGS, and Stan. Academic Press.
McConkie, G. W., Kerr, P. W., Reddix, M., \& Zola, D. (1988). Eye movement control during reading: I. the localisation of initial eye fixations in words. Visual Research, 28(10), 1107-1118. https://doi.org/10.1016/0042-6989(88)90137-X

McConkie, G. W., \& Rayner, K. (1975). The span of the effective stimulus during a fixation in reading. Perception Psychophysics, 17(6), 578-586.

McDonald, S. A., Carpenter, R., \& Shillcock, R. C. (2005). An anatomically constrained, stochastic model of eye movement control in reading. Psychological Review, 112(4), 814.

Nuthmann, A., Engbert, R., \& Kliegl, R. (2005). Mislocated fixations during reading and the inverted optimal viewing position effect. Vision Research, 45(17), 2201-2217.

O'Regan, J. K., \& Lévy-Schoen, A. (1987). Eye-movement strategy and tactics in word recognition and reading. In Coltheart, M. (Ed.) Attention and performance xii: The psychology of reading ( $p$. 363-383). Hillsdale: Lawrence Erlbaum Associates, Inc.

Plummer, M. (2016). rjags: Bayesian graphical models using MCMC [Computer software manual]. Retrieved from https://CRAN. $\mathrm{R}$-project.org/package=rjags ( $\mathrm{R}$ package version 4-6).

Radach, R., Huestegge, L., \& Reilly, R. (2008). The role of global top-down factors in local eye-movement control in reading. Psychological Research, 72(6), 675-688.

Radach, R., \& McConkie, G. W. (1998). Determinants of fixation positions in words during reading. Eye Guidance in Reading and Scene Perception, 77-100. https://doi.org/10.1016/B978-008043361-5/ 50005-5.

Rayner, K. (1979). Eye guidance in reading: Fixation locations within words. Perception, 8(1), 21-30.

Rayner, K. (1998). Eye movements in reading and information processing: 20 years of research. Psychological Bulletin, 123(3), 372-422. https://doi.org/10.1037/0033-2909.124.3.372.

Reichle, E. D., Rayner, K., \& Pollatsek, A. (2003). The E-Z Reader model of eye-movement control in reading: Comparisons to other models. Behavioral and Brain Sciences, 26(4), 445-476.

Reilly, R. G., \& O'Regan, J. K. (1998). Eye movement control during reading: A simulation of some word-targeting strategies. Vision Research, 38(2), 303-317. https://doi.org/10.1016/S0042-6989 (97)87710-3.

Reilly, R. G., \& Radach, R. (2006). Some empirical tests of an interactive activation model of eye movement control in reading. Cognitive Systems Research, 7(1), 34-55.

Schad, D. J., \& Engbert, R. (2012). The zoom lens of attention: Simulating shuffled versus normal text reading using the swift model. Visual Cognition, 20(4-5), 391-421.

Schotter, E. R., Bicknell, K., Howard, I., Levy, R., \& Rayner, K. (2014). Task effects reveal cognitive flexibility responding to frequency and predictability: Evidence from eye movements in reading and proofreading. Cognition, 131(1), 1-27.

Underwood, G., \& Radach, R. (1998). Eye guidance and visual information processing: Reading, visual search, picture perception and driving. In: Eye guidance in reading and scene perception (pp. 1-27). Elsevier.

Vonk, W., Radach, R., \& van Rijn, H. (2000). Eye guidance and the saliency of word beginnings in reading text. In: Reading as a perceptual process (pp. 269-299). Elsevier.

Publisher's note Springer Nature remains neutral with regard to jurisdictional claims in published maps and institutional affiliations. 